\title{
De l'Empire ottoman à la République : la caricature turque sous l'influence du français
}

Füsun Ataseven, Emine Bogenç Demirel et Elif Ertan

\section{(2) OpenEdition \\ Journals}

Édition électronique

URL : https://journals.openedition.org/dhfles/347

DOI : $10.4000 /$ dhfles.347

ISSN : 2221-4038

Éditeur

Société Internationale pour l'Histoire du Français Langue Étrangère ou Seconde

Édition imprimée

Date de publication : 1 janvier 2007

Pagination : 260-272

ISSN : 0992-7654

Référence électronique

Füsun Ataseven, Emine Bogenç Demirel et Elif Ertan, « De l'Empire ottoman à la République : la caricature turque sous l'influence du français », Documents pour l'histoire du français langue étrangère ou seconde [En ligne], 38/39 | 2007, mis en ligne le 14 décembre 2010, consulté le 27 mai 2021. URL http://journals.openedition.org/dhfles/347 ; DOI : https://doi.org/10.4000/dhfles.347

Ce document a été généré automatiquement le 27 mai 2021.

(c) SIHFLES 


\title{
De l'Empire ottoman à la République : la caricature turque sous l'influence du français
}

\author{
Füsun Ataseven, Emine Bogenç Demirel et Elif Ertan
}

1 La tolérance est le signe distinctif de l'humour. L'humour a besoin de tolérance pour naître. Depuis toujours l'humour et la tolérance se nourrissent mutuellement. L'un crée l'autre ou l'un provoque l'autre. L'humour est présent sous différentes formes: histoires drôles, poèmes drôles, caricatures, marionnettes, pièces comiques...

Dans la société, il existe des pressions sur la vie sociale, économique, politique et sexuelle. C'est grâce à l'humour que la pression laisse place à la tolérance. Dans la caricature, les symboles se présentent dans une logique humoristique et se placent dans des attitudes et des mouvements inhabituels.

3 La caricature est un art comme les autres, créé pour exprimer un problème, pour critiquer une idée ou pour proposer une solution par l'intermédiaire de l'humour. Elle peut se présenter sous forme d'un corps humain mal proportionné, sous forme de visages d'hommes et de femmes transformés en portraits abstraits, ou sous la forme de dessins concrets racontant des histoires drôles.

4 La caricature se fait surtout connaître par le biais de la presse écrite. Aussi, les péripéties que subit la presse influencent-elles directement le développement de la caricature. Sur les caricatures, des situations impossibles deviennent réelles. Les frontières entre les classes sociales disparaissent et un homme politique très connu peut apparaître tout à coup à côté d'un homme de la rue et discuter de problèmes réels.

5 La caricature est une forme écrite de l'humour. En Turquie, son apparition prend un certain temps et reste sous l'influence des pays occidentaux, la France surtout. Bien que l'humour soit très ancien, la caricature, qui se fonde sur le dessin, progresse assez tardivement dans une société musulmane.

6 L'humour, qui apparaît en général comme une expression culturelle sous les régimes totalitaires, est né à l'époque du Sultan Abdülhamid II. Dans la civilisation ottomane, 
nous pouvons citer pour la caricature, des antécédents comme les miniatures, le théâtre d'ombres traditionnel turc Karagöz. Depuis les années de Tanzimat jusqu'à la République, sous l'influence de la culture française, l'humour oral a été remplacé par l'humour écrit.

Le bagage culturel des caricaturistes était spécialement nourri de l'humour oral. Les œuvres classiques de l'humour occidental n'étaient pas encore traduites. Bien que les écrivains de l'époque connaissaient des langues étrangères, ce n'était pas toujours suffisant pour acquérir la langue humoristique. [...] Tout comme le roman qui a été importé de l'Occident pour contribuer à notre effort de modernisation, la caricature turque a commencé à l'époque de Tanzimat. (Çeviker, T., 1985, p. 20)

7 C'est ainsi que l'humour étranger nous a apporté la diversité et la qualité. Dans notre recherche, nous essayerons de montrer l'influence du français sur la caricature à l'époque du passage de l'Empire ottoman à la République, dans le cadre des sujets tels que la relation entre homme et femme, la politique, l'argent... Nous nous focaliserons plus particulièrement sur les œuvres des caricaturistes qui ont vécu en France, comme Teodor Kasap, Nişan Berberyan, Ali Fuat Bey et, dans ce contexte, sur les revues de l'époque comme Diyojen, Terakki Eğlencesi, Çaylak, Çıngıraklı Tatar.

\section{Les caricatures turques jusqu'au $\mathrm{XIX}^{\mathrm{e}}$ siècle}

8 En Turquie, vers le XIII ${ }^{e}$ siècle, l'humour oral crée les histoires de Nasreddin Hoca. Sa renommée va de pays en pays, de siècles en siècles avec ses histoires courtes et morales. Pendant sa vie, il a voulu montrer la juste voie au peuple par l'intermédiaire de ses aventures humoristiques. Vers le XIV ${ }^{e}$ siècle, le théâtre d'ombres traditionnel turc donne naissance aux histoires drôles de Karagöz-Hacivat.

En faisant des recherches sur l'humour et les caricatures, nous avons trouvé des exemples de miniatures. Même si au départ, cet art n'a pas comme but de faire de l'humour, vers le $\mathrm{XVI}^{\mathrm{e}}$ siècle, ces dernières constituent les premiers exemples de caricatures écrites en tant qu'éléments précurseurs.

$\mathrm{Au}$ début, durant une certaine période, on dessinait sur les murs des cafés des esquisses sous forme d'histoires continues. Ces lieux sont très vite devenus à la mode. Voici un exemple de miniature d'un type assez connu qui se présente sous forme de devinette. Les dessins sont primitifs, explicatifs et bien alignés. En voyant les dessins, on comprend l'axe principal de l'histoire. 


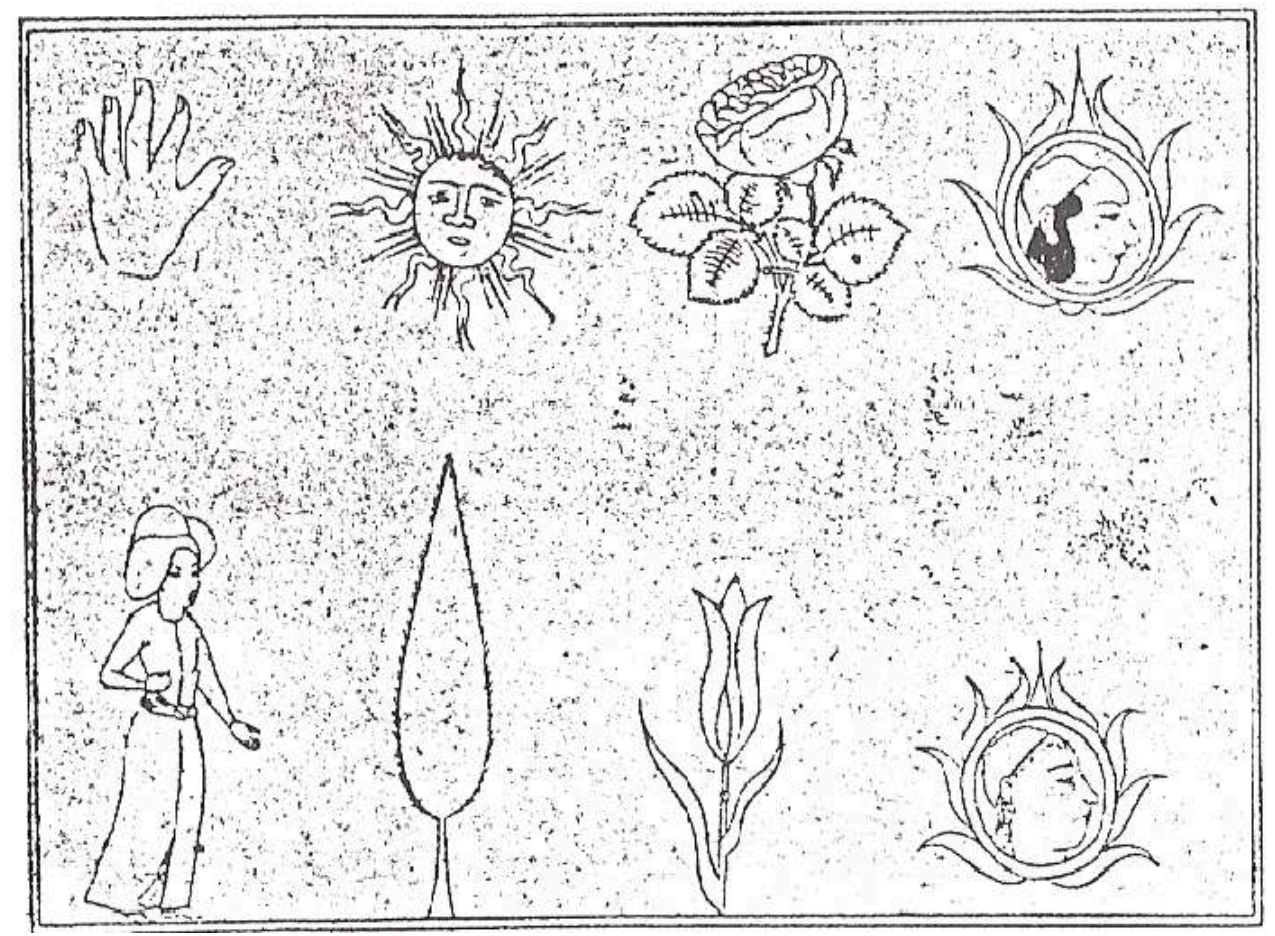

Buharalı Abdullah, Palais Topkapı, Galerie de la peinture.

(Koloğlu, O., 2005, p. 12)

11 À l'écrit, l'histoire se présente sous forme de deux vers :

\begin{tabular}{|l|l|}
\hline Pençe-i âfitab-1 gül'ü ruhsar & La fille aux mains de soleil et aux joues en rosées \\
\hline \hline Dilberi servikad'i lâle iza & Le jeune homme aux joues de tulipe et à la taille élancée \\
\hline
\end{tabular}

Dans l'Empire ottoman, avec le passage à la période de Tanzimat, l'humour oral des siècles précédents laisse la place à l'humour écrit. Sous l'influence des pays occidentaux, de la France en particulier, ce nouveau genre apparait dans les journaux et les revues sous forme d'anecdotes, d'histoires courtes et de caricatures.

Diyojen, la première revue d'humour, éditée par Hovsep Vartanyan, voit le jour en 1852. Sa première caricature est publiée en 1871. Avec la déclaration de la Constitution, la libéralisation qui se montre dans les caricatures provoque de vives discussions au Parlement, présidé par le Sultan lui-même.

14 Avant même l'apparition de la première revue humoristique, le premier code pénal prévoit la censure des journaux d'humour. Ce code est la traduction du code pénal français :

(Article 139) Celui qui publie ou fait publier des dessins et des descriptions contre les habitudes/coutumes du peuple ou fait des critiques dans les articles/poèmes doit être puni en payant entre 1-5 mecidiye en or et doit être emprisonné pour une durée de 24 heures à une semaine. (Çeviker, T., 1985, p. 61)

15 Ces dispositions créent des journalistes espions, mais, évidemment, face à ces personnages, les autres journalistes réagissent sous des formes diverses, comme les 
pages blanches, les lettres au public, les caricatures des espions... Voici deux exemples de réactions à la censure provenant des journaux Hayal et Karagöz.

Une page blanche du journal Karagöz avec une simple phrase en bas de la page.

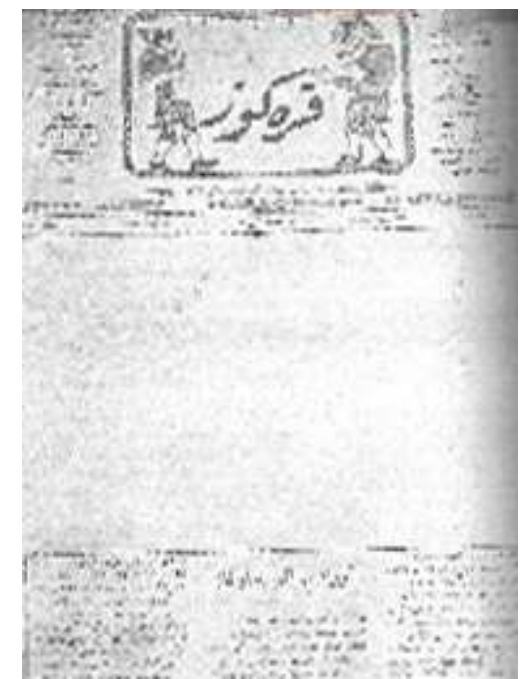

Nous mettions sur cette page en général une caricature. Et celle-ci nécessitait beaucoup d'argent. Maintenant ce moyen est impossible. Ceci n'est pas trop mal, puisque d'une certaine façon nous avons économisé de l'argent. Nous avons toujours nos caricatures, mais pas de permission. Si nous l'obtenons, nous les publierons. Si nous les publions, vous les verrez. Si vous les voyez, vous serez contents. Si vous êtes contents, vous achèterez de nouveau Hayal. Si vous achetez Hayal, vous prendrez plaisir. Ainsi de suite... Et voilà, nous avons fait de notre mieux, nous n'avons pas laissé de page blanche. S'il y a encore des vides, c'est votre faute, ne restez pas silencieux, remplissez les vides en nous écrivant.

(Koloğlu, O., 2005, p. 186)

Dans la première revue de caricatures Diyojen, Namık Kemal parle d'un homme avec des oreilles d'âne pour dénoncer les espions. Et aussitôt, "les grandes oreilles» apparaissent. Nos recherches nous conduisent au propriétaire de ces oreilles, Garabet Panosyan, journaliste, éditeur et parlementaire. Il fait souffrir les écrivains de l'époque. Les caricaturistes de son temps se vengent en le caricaturant. 


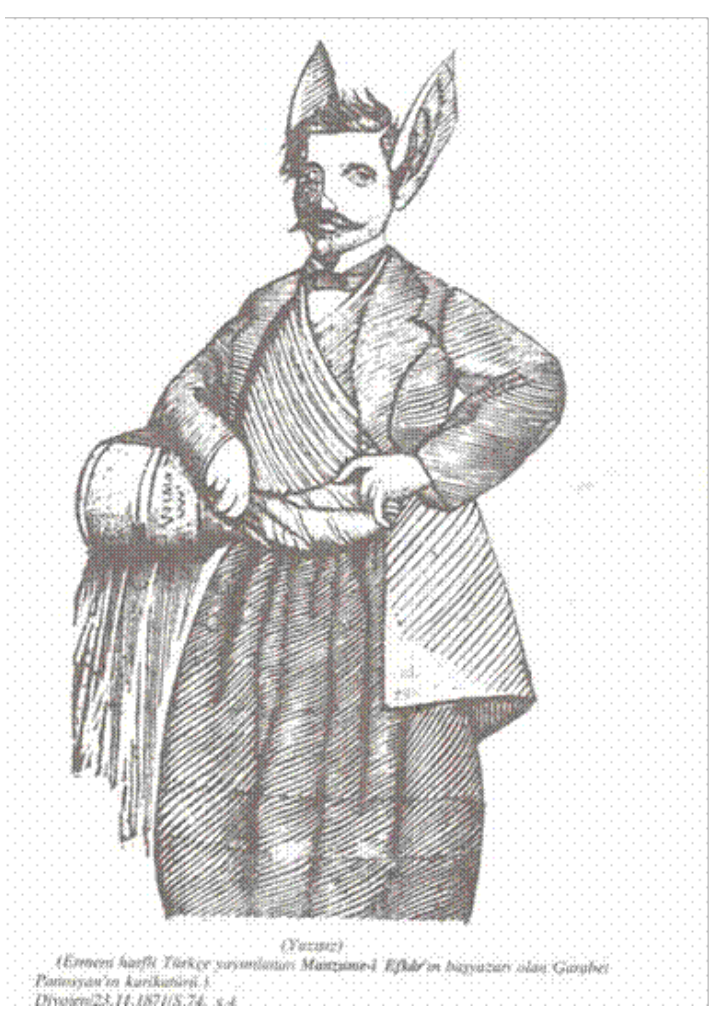

Pourquoi chercher des espions aux grandes oreilles puisqu'on a des journaux avec leurs grandes gueules fidèles au gouvernement?

(Çeviker, T., 1987, p. 147)

arménien et en français. Les textes étaient traduits le plus souvent du français. Namık Kemal et Teodor Kasap, deux auteurs très connus, ont, comme plusieurs d'entre eux, suivi des études en France. Ils sont restés sous l'influence très nette de la culture française et des auteurs français. Le caricaturiste très connu de l'époque, Santr, d'origine française, publiait dans Hayal et est connu pour ses caricatures sur les modes de vie.

Les caricaturistes de l'époque de Tanzimat étaient tellement prolifiques qu'ils ne cessaient de traiter les sujets les plus variés. Les relations familiales entre les hommes, les femmes et les enfants sont les thèmes les plus représentés : la vie familiale, les maris qui essaient de s'adapter au nouveau mode de vie, les enfants gâtés qui, eux, s'adaptent très vite à la vie occidentale et qui ne sont plus comme les enfants obéissants d'autrefois, le docteur qui flirte avec ses malades... Par ailleurs, le changement de vêtements et de coiffures provoque jalousie et disputes entre mari et femme. Les hommes ont du mal à accepter la nouvelle apparence de leur femme. 


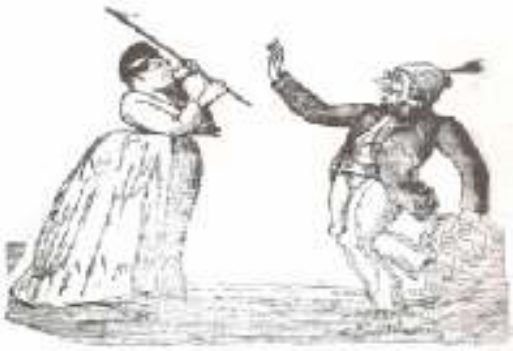

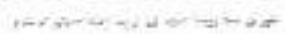

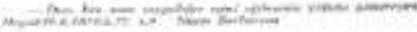

- Il est temps que je t'apprenne comment faire de toi un mari respectueux !Hayal (Çeviker, T., 1987, p. 216)

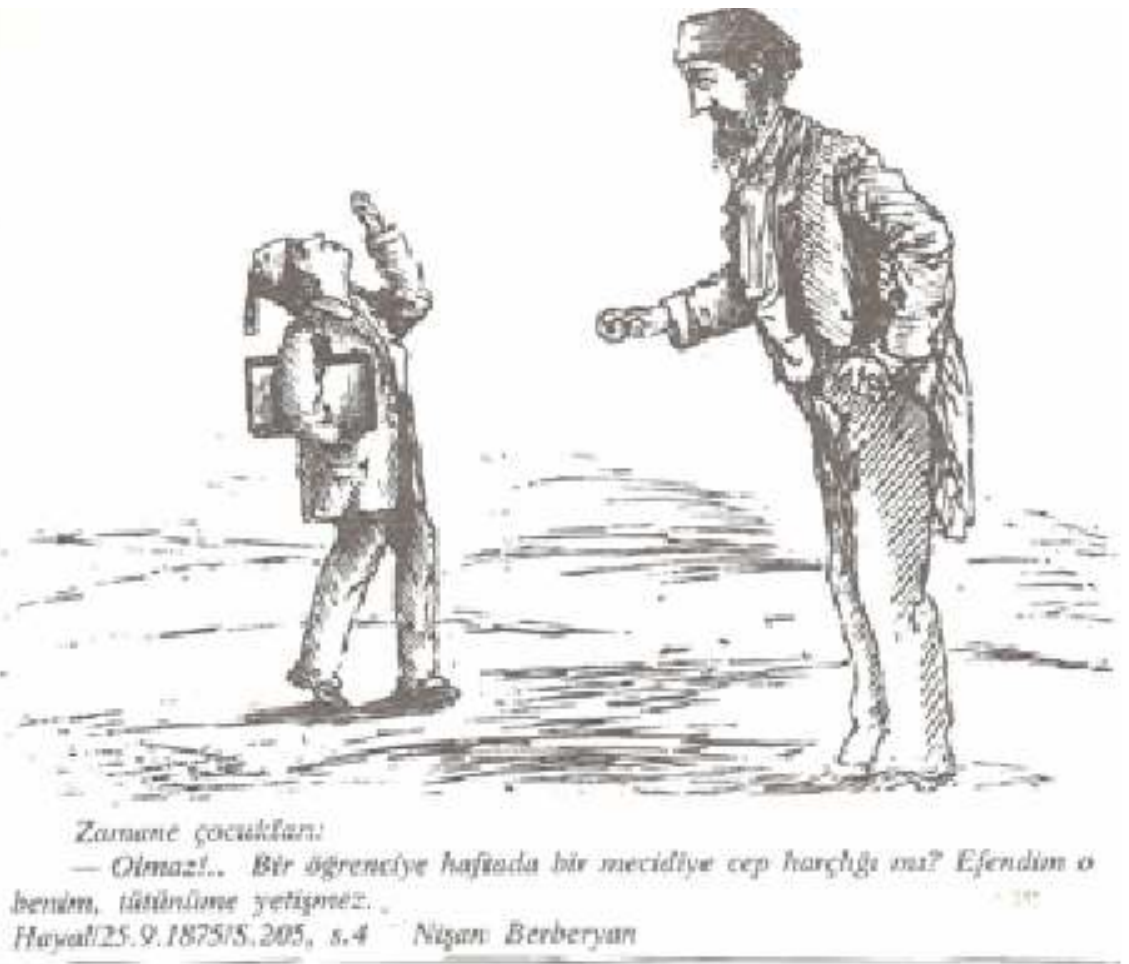

- Non, ce n'est pas possible un "mecidiye" par semaine comme argent de poche? Ce n'est même pas suffisant pour mon tabac. Hayal

(Çeviker, T., 1987, p. 159)

21 L'influence du mode de vie à la française est aussi un thème de prédilection. Parmi les premières caricatures, les «Femmes de Pera » occupent une place importante. Les habits et les manières de vivre des femmes de Pera, quartier d'Istanbul habité autrefois par les Européens, sont assez différents de ceux des autres femmes. La comparaison des deux mondes par le dessin humoristique est alors très à la mode. 


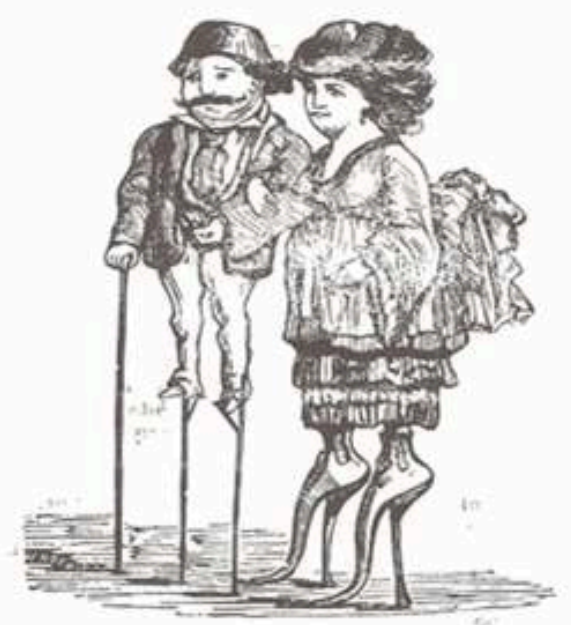

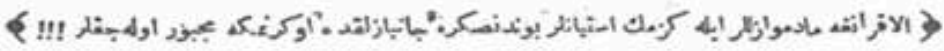

Alafranga matmazeller ile gezmek isteyenter, bundan sonna cambazhk da ogrenmeye zorunlu olacaklar!I!

Tiyatro / 9.5.1874/ S. 12, 5. 4 Nigan Berberyan

Ceux qui souhaitent sortir avec les demoiselles à la française doivent être acrobates. Tiyatro.

(Çeviker, T., 1987, p. 40)

Quant à l'influence de la langue, nous remarquons souvent l'utilisation de mots français dans l'écriture turque ; le premier mot français utilisé par les humoristes pendant cette période est le mot «civilisation » écrit tel qu'il est prononcé en turc : « sivilizasyon ». La manière de s'habiller, de s'exprimer ou de penser traduisent le fait d'être « civilisé ». La France et Paris se font sentir dans la vie quotidienne.

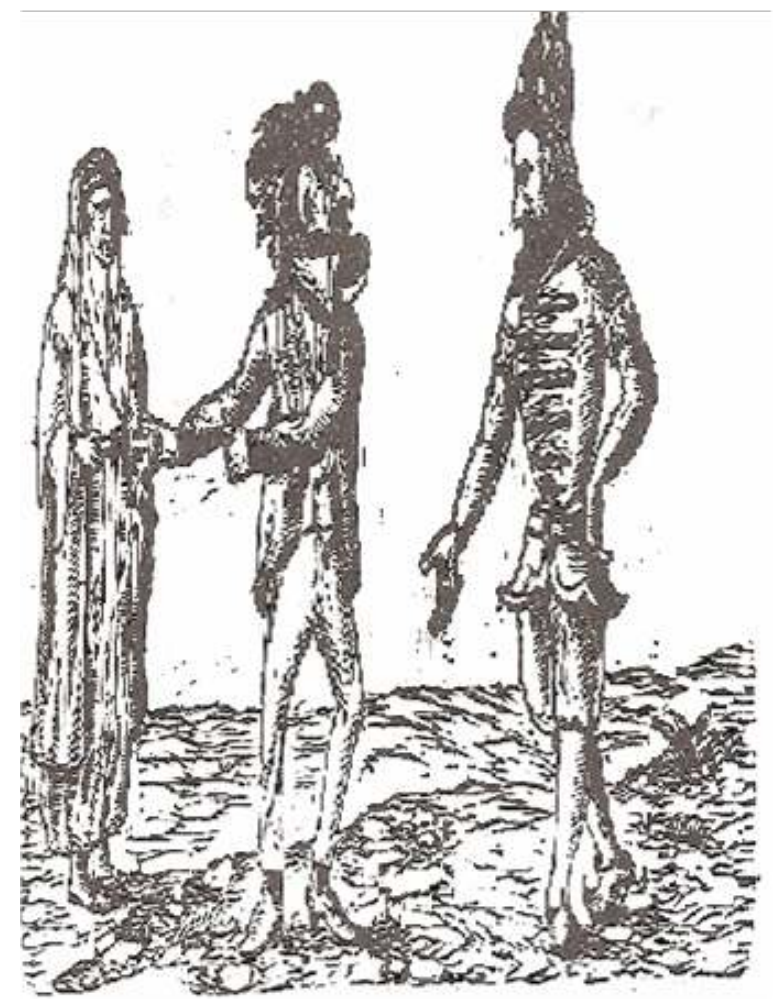


- Mon cher, je te présente ma sœur.

- Qu'est-ce que tu fais?

- Je te " prezante "... C'est la mode mon cher. Hayal

(Çeviker, T., 1987, p. 214)

23 Les caricaturistes de cette époque sont aussi des philosophes de leur temps comme Teodor Kasap, Agop Baronyan, Zakarya Beykozluyan, Mehmet Tevfik... Dans la tristesse de Berberyan, le monde féminin de Santr, les critiques politiques de Baronyan, les critiques sociales de Mehmet Tevfik, nous voyons toujours l'influence de la pensée française. Avec le temps, les sujets se multiplient, et même les problèmes municipaux apparaissent. L'eau sale, la poussière des rues, les mauvaises odeurs sont les principaux sujets des caricatures de l'époque de Tanzimat.

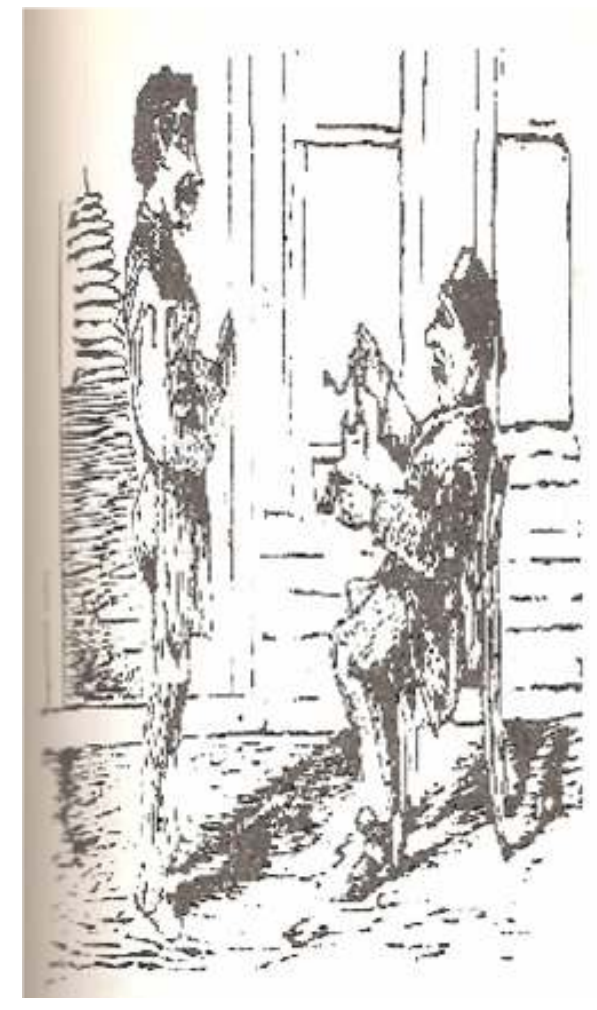

- Serveur! Il y a une grenouille dans mon café ?

- Eh ! Monsieur, qu'attendiez vous d'autre d'un café préparé avec l'eau de la municipalité de Terkos ? Tiyatro (Çeviker, T., 1985, p. 51)

24 Abdülhamid était un personnage très proche de l'art et très ouvert à l'adoption des manières européennes à la vie quotidienne ottomane. Il réalisait lui-même des sketchs qui critiquaient ses proches dans le sérail. Mais il ne supportait pas les critiques des autres sur lui-même, il était contre la liberté de la discussion, la liberté de la presse et finalement contre l'humour libre.

Selon la coutume, personne n'osait critiquer le Sultan considéré comme sacré en tant que chef de la religion. Avec la libre entrée des idées révolutionnaires, le nombre des revues d'humour se multiplie. Le héros de ces caricatures est une fois sur deux Abdülhamid. Pendant cette période des idées de la Révolution, de la Constitution et de la République, il reste le Sultan absolu. Bien que Abdülhamid ait installé la Constitution et le Parlement, c'est lui-même qui interdit les publications de ces caricatures. Donc pendant 
son règne, on trouve dans toutes les revues des caricatures du Sultan avec son nez immense que personne n'avait droit de mentionner.

Sous l'influence des idées de la Révolution Française, dans les caricatures de cette époque, le symbole de la démocratie est une belle femme ; le symbole du despotisme est Abdülhamid.

Les années de censure du Sultan Abdülhamid

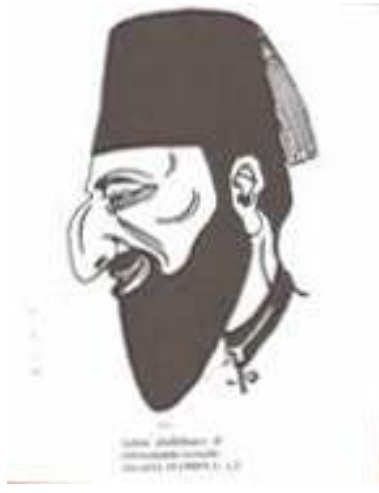

Est-ce par peur de la critique ou bien par honte de son nez?

(Çeviker, T., 1987, p. 289)

Après le règne d'Abdülhamid, la caricature turque demeure aux ordres des partis politiques. Vers la fin de l'Empire ottoman, l'unique parti politique, İttihat Terakki, soutenait ouvertement les caricaturistes aux idées révolutionnaires comme Cem et sa revue d'humour Kalem.

\section{Les caricatures à la fin du $\mathrm{XIX}^{\mathrm{e}}$ et au début du $\mathrm{XX}$ siècle}

Avant la grande Guerre d'Indépendance, la diffusion des caricatures passe de la main des Tarikat (ordres religieux) à l'ordre gouvernemental. Le Sultan était soutenu par la revue Aydede, le gouvernement parlementaire créé à Ankara était soutenu, quant à lui, par la revue d'humour Güleryüz.

Entre 1923 et 1928, le thème principal est l'ambiance créée par la victoire de la Guerre d'Indépendance. Les réformes sociales, économiques et politiques influencent les revues d'humour. Tous les artistes montrent par leurs dessins une grande ouverture d'esprit: de nouvelles formes apparaissent où nous pouvons voir qu'ils s'adaptent facilement à la société moderne et laïque.

Après 1923, la réforme de l'alphabet va jouer un rôle important pour la vie humoristique en Turquie. Avec le nouvel alphabet, les règles anciennes changent et l'utilisation des expressions, des mots ainsi que les règles de grammaire de la langue turque sont différentes. Les phrases deviennent courtes et simples. Cette réforme a aussi beaucoup transformé le visage de la caricature. La manière de dessiner se modifie et une nouvelle forme de liberté envahit les revues. Les dessins, les histoires et les jeux de mots subissent également des changements grâce aux nouveaux paradigmes de la jeune République turque. 
Comment on gagne sa vie. Aydede

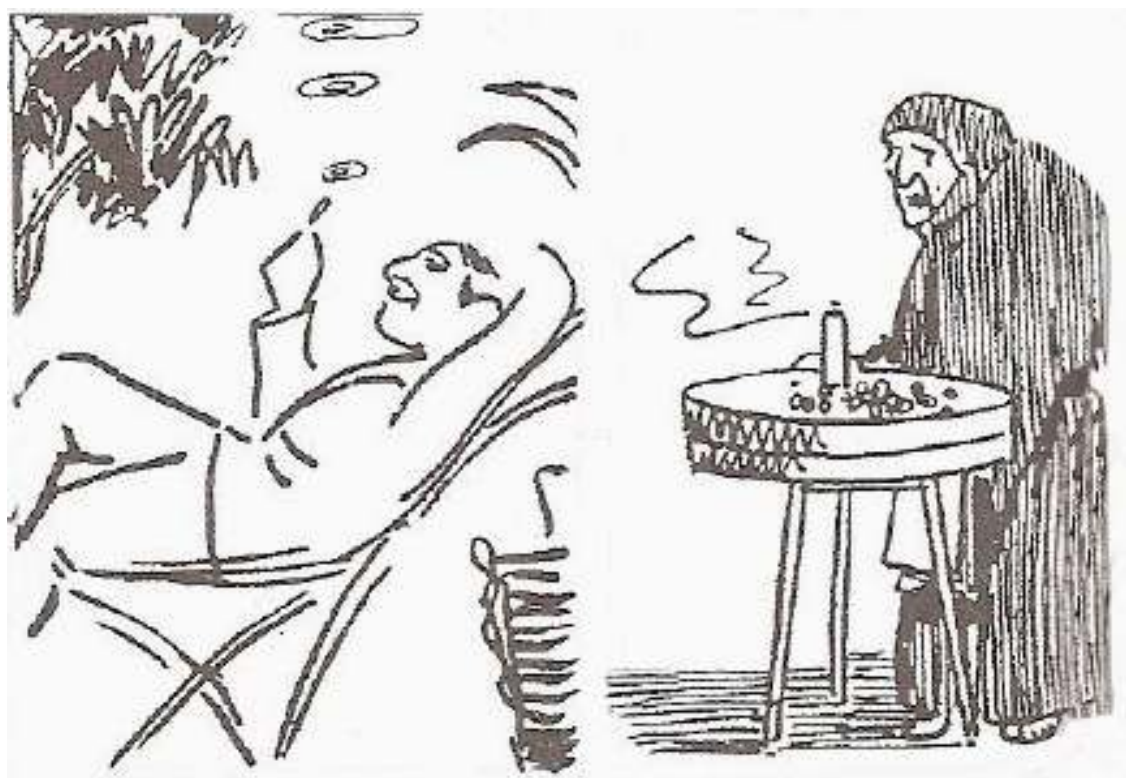

(Rentier de la guerre) I (Femme immigrée)

Éloge d'Atatürk après la Guerre d'Indépendance Güleryüz

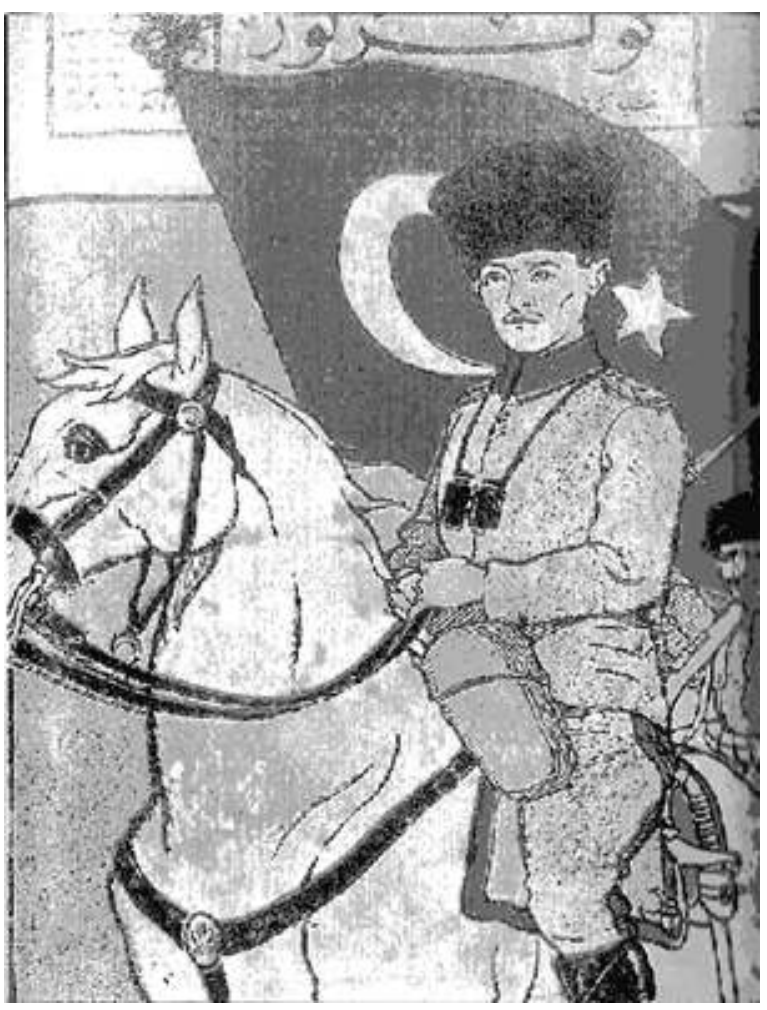

(Koloğlu, O., 2005, p. 202, 234)

31 La revue Güleryüz est la seule revue qui soutienne le nouveau gouvernement d'Ankara. Elle est fondée par les célèbres caricaturistes Sedat Simavi et Yusuf Ziya.

32 Aydede, qui est une ancienne revue publiée à Istanbul, est diffusée par plusieurs caricaturistes et auteurs déjà très connus. Dans cette revue, il y a des caricaturistes 
français et la moitié de la revue est rédigée en français. On y trouve des caricatures contre Atatürk, contre l'armée du nouveau gouvernement. Les célèbres caricaturistes sont Ramiz, Cem, Ratip Tahir, Rıfkı, Münif Fehim, etc.

Après la guerre, pendant la Nouvelle République turque, et jusqu'en 1930, la Turquie découvre des caricaturistes connus par leurs journaux et par leurs revues qui diffusent des caricatures plutôt engagées. Parmi les sujets principaux traités par les artistes, mises à part les critiques politiques, il y a le «mode de vie », «Pera ", la "liberté des femmes » et les habitudes tragi-comiques des « nouveaux riches ».

L'influence de la culture française se fait toujours sentir dans les formes et les traits des dessins de l'époque, avec les caricaturistes comme Ahmet Rıfkı, Cemal Nadir Güler et bien sûr Sedat Simavi.

« Les néo-riches », Güleryüz, Sedat Simavi

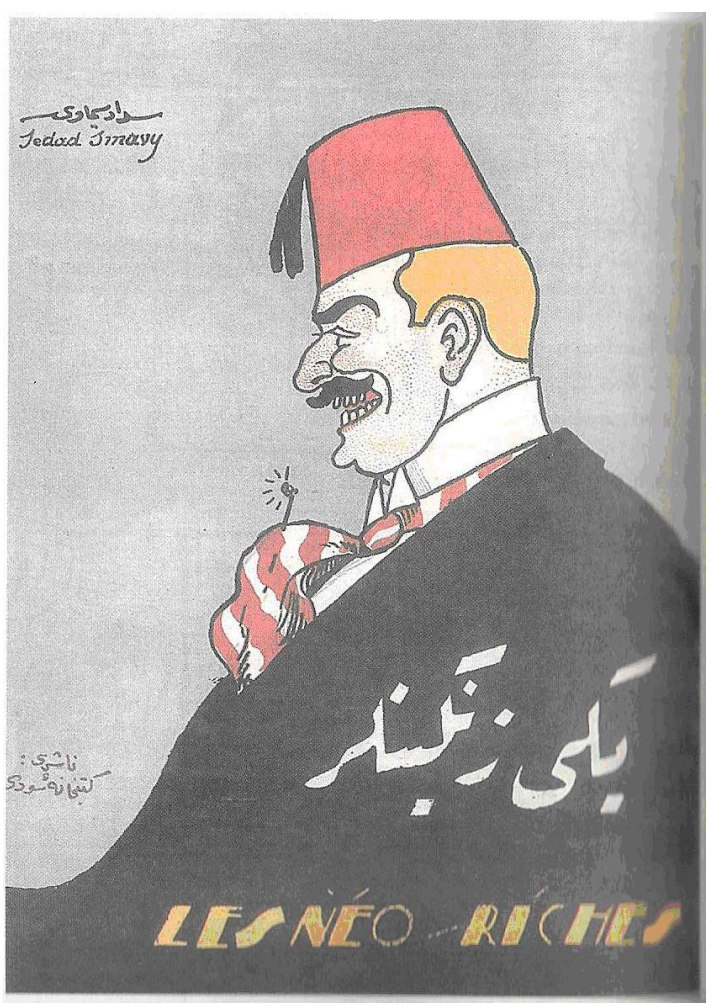

(Koloğlu, O., 2005, p. 234)

La vie culturelle de la nouvelle génération est très animée, riche et libre. Tout est discutable, tout est critiquable, sauf la laïcité, qui est tout à fait nouvelle et est à la base de la Nouvelle République.

Le tirage des revues d'humour pendant cette période se multiplie d'une façon étonnante.

Nous finirons notre propos en rappelant les mots de Macit Efendi lors des discussions à l'Assemblée nationale (Meclis-i Mebusan) en 1877 :

En ce qui concerne les journaux d'humour, tous les pays ont toujours montré une grande tolérance envers cette importante et spécifique branche de la presse. Les journaux d'humour sont la mesure de la clarté d'opinion, de la finesse, des sentiments, de la politesse, en un mot de «l'intelligence» d'un pays. C'est un art dur à pratiquer. Il est tellement difficile de se moquer sans être vulgaire, tellement 
difficile de dire des choses risibles sans être agressif ! [...] S'il est impossible de rester indifférent face à une agression grossière et humiliante, s'il est aussi impossible de rester indifférent face à des fines critiques non vulgaires, il est tout aussi impensable de se révolter face à de fines critiques non vulgaires. » (Cité par Çeviker, T., 1968, pp. 60-64)

\section{BIBLIOGRAPHIE}

BALCIOĞLU, S. (1999), Cumhuriyetin 75. Yılında Türk Karikatürü, Istanbul : İş bankası Kültür Yayınları.

ÇEVIKER, T. (1985), Ali Fuat Bey, Osmanlı Tokadı, Istanbul : Adam Yayınları.

ÇEVIKER, T. (1985), Nisan Berberyan, Terakki edelim beyler, Istanbul : Adam Yayınları.

ÇEVIKER, T. (1987), Gelişim sürecinde Türk Karikatürü Tanzimat Dönemi, Istanbul : Adam Yayınları.

KABACALI, A. (2001), Semih Balcıoğlu, Istanbul : Tüyap Fuarcılık A.ş.

KOLOĞLU, O. (2005), Türkiye Karikatür Tarihi, Istanbul :Bileşim Yayınevi.

URAL, Y. (1984), Cemal Nadir karikatür albümü, Istanbul : Milliyet Yayınları.

\section{RÉSUMÉS}

L'humour, qui apparaît en général comme une expression des cultures sous les régimes totalitaires, est né dans la culture turque à l'époque du Sultan Abdülhamit II. Des années de Tanzimat jusqu'à la République, sous l'influence de la culture française, l'humour oral a été remplacé par l'humour écrit. C'est ainsi que l'humour étranger nous a apporté la diversité. Dans notre recherche, nous essayons de montrer l'influence du français sur la caricature à l'époque de transition de l'Empire ottoman à la République, dans le cadre des sujets tels que la relation entre homme et femme, la politique, l'argent... en nous focalisant plus particulièrement sur les œuvres des caricaturistes qui ont vécu en France.

Humour, which could be regarded as an expression of cultures oppressed by totalitarian regimes, emerged in the Turkish culture during the reign of Abdülhamit the Second. Written humour, along with verbal humour coming from the Tanzimat Period to the Republic, had gained importance with the influence of the French culture; therefore, foreign humour offered diversity to the Turkish culture. In our research, we would like to dwell on the French influence on Turkish caricature through transition period from the Ottoman Empire to the Turkish Republic, particularly focusing on the concepts like man-woman relationship, politics, money... and the works of the caricaturists who had lived in France. 
INDEX

Mots-clés : caricature, culture française, culture turque, humour, régimes totalitaires

Keywords : caricature, French culture, humour, totalitarian regimes, Turkish culture

\section{AUTEURS}

\section{FÜSUN ATASEVEN}

Université technique de Yıldız, Département d'Interprétation et de Traduction, Turquie EMINE BOGENÇ DEMIREL

Université technique de Yıldız, Département d'Interprétation et de Traduction, Turquie

\section{ELIF ERTAN}

Université technique de Yıldız, Département d'Interprétation et de Traduction, Turquie 